Check for updates

Cite this: RSC Adv., 2019, 9, 19630

\title{
Ordered structure effects on $\beta$-nucleated isotactic polypropylene/graphene oxide composites with different thermal histories
}

\author{
Yansong $Y u^{a}{ }^{a}$ Ruizhang $X u{ }^{a}$ Jinyao Chen, (D) a Jian Kang, (D) *a Ming Xiang, ${ }^{a}$ \\ Yuanjie $\mathrm{Li}^{\text {*b }} \mathrm{Lu} \mathrm{Li}^{\mathrm{c}}$ and Xingyue Sheng ${ }^{\mathrm{C}}$
}

In this paper, the influence of ordered structure effects (OSE) on crystallization behaviors of $\beta$-nucleated isotactic polypropylene/graphene oxide ( $\beta$-iPP/GO) composites with different thermal histories, which crystallized at a slow cooling rate (called SLOW), fast cooling rate (called FAST) and medium cooling rate (called MED), respectively, was studied by differential scanning calorimetry (DSC) and wide-angle X-ray diffraction (WAXD). The ordered structure status of three samples before crystallization measurement was controlled by tuning the fusion temperature $T_{\mathrm{f}}$ and melting time $t_{\mathrm{m}}$. The results showed that for all samples, OSE would occur in an appropriate $T_{\mathrm{f}}$ region (Region II). The OSE efficiency of MED was the highest, while that of SLOW were the lowest. It was also found that the crystallinity and crystalline perfection of SLOW were the highest, while those of FAST were the lowest. The effects of the melting time $t_{m}$ on the OSE were also investigated. At $T_{f}=172{ }^{\circ} \mathrm{C}$, the OSE efficiency of FAST reached the maximum at $t_{\mathrm{m}}=5 \mathrm{~min}$, while that of SLOW reached the maximum at $t_{\mathrm{m}}=20 \mathrm{~min}$. It was indicated that the OSE efficiency was affected by thermal history, and it could be improved by selecting the appropriate $t_{\mathrm{m}}$. Related mechanisms concerning the roles of thermal history on the OSE behavior were proposed based on the results of DSC and in situ SAXS.

Received 7th May 2019

Accepted 18th June 2019

DOI: 10.1039/c9ra03416a

rsc.li/rsc-advances
iPP/GO composites with high mechanical and high barrier properties could be prepared. ${ }^{3}$

iPP is a kind of semi crystalline resin with five crystalline structures: $\alpha$-form, $\beta$-form, $\gamma$-form, $\delta$-form and smectic phase. $^{16,17} \beta$-form, as a metastable phase, can only form under special conditions as follows: crystallization of melt subject to shear or elongate, directional crystallization in specific temperature gradient, crystallization after quenching to a certain temperature range, or crystallization in the presence of $\beta$-nucleating agents ( $\beta$-NA) ${ }^{18,19}$ The addition of $\beta$-NA is the most effective way for the formation of high relative content of $\beta$ phase, which increases the toughness while decreases the stiffness. On the contrary, $\alpha$-nucleating agent $(\alpha-\mathrm{NA})$ induces the formation of $\alpha$-phase, which strengthens the stiffness while decreases the toughness. ${ }^{20}$ Unfortunately, studies have shown that GO acts as an $\alpha$-NA during the iPP crystallization, which prevents the formation of high amount of $\beta$-phase. ${ }^{21}$ Therefore, the key issue is how to balance the stiffness and toughness of iPP/GO composites.

It may be an effective method to substantially promote the iPP $\beta$-crystallization by the formation of pre-ordered melt structure in $\beta$-nucleated iPP melt. It has been shown that polymer crystallization is a process from completely molten to melt pre-ordered then to completely crystallization, which is quite different from the crystallization of small molecule..$^{22,23}$ There will be an amount of ordered structure remaining in the 
melt when the fusion temperature $T_{\mathrm{f}}$ is not high enough to melt the iPP entirely. During subsequent crystallization, ordered structure can evidently increase the nucleation density, decrease the crystal size, accelerate the crystal growth rate, and even affect the polycrystalline behavior, which is called as the melt memory effect, self-nucleation, or Ordered Structure Effect (OSE)..$^{24,25}$

Although the physical properties of ordered structure are not yet clear, it can be determined that the existence of ordered structure is beneficial to accelerate the crystallization rate and increase the crystallization temperature. In addition, recent studies have shown that the ordered structure is crucial for the formation of $\beta$-phase. Yan et al. ${ }^{26}$ added partially melting iPP fiber into the supercooled iPP melt and found that the partially orientated and molten ordered structure obtained from the iPP fiber played an extremely important role in the iPP $\beta$-nucleation. Alfonso et al. ${ }^{27}$ found that applying shear flow to iPP melt and the introduction of partially molten fiber had similar effects on the formation of $\beta$-phase. Shen and Zheng et al. ${ }^{28-32}$ studied the crystallization behavior of iPP melt under low shear stress and found that only in the existence of the ordered structure, $\beta$ phase would be able to form in the sheared iPP melt.

Recently, our group found that for composites of iPP/GO nucleated by $\beta$-nucleating agent (tradename WBG-II) (GO : WBG-II $=0.5 \mathrm{wt} \%: 0.05 \mathrm{wt} \%$ ), the ordered structure was favorable for the formation of $\beta$-phase. ${ }^{33}$ According to the thermal treatment in Fig. 1, the ordered structure status in the melt could be controlled by tuning the fusion temperature $T_{\mathrm{f}}$. The results showed that the entire range of $T_{\mathrm{f}}$ could be divided into three regions. In Region $\mathrm{I}\left(T_{\mathrm{f}}>178^{\circ} \mathrm{C}\right)$, no ordered structure existed, and the nucleation efficiency $\mathrm{NE}$ of GO was obviously higher than that of WBG-II, resulting in no formation of $\beta$ phase. In Region II $\left(T_{\mathrm{f}}=168-178{ }^{\circ} \mathrm{C}\right)$, an amount of ordered structure existed in the melt, resulting in substantially increase of the relative content of $\beta$-phase $\beta_{\mathrm{c}}$ (achieving the maximum of $85.8 \%$ ), indicating that the ordered structure had a strong promoting effect on the $\beta$-crystallization of $\mathrm{iPP} / \mathrm{GO} / \mathrm{WBG}-\mathrm{II}$

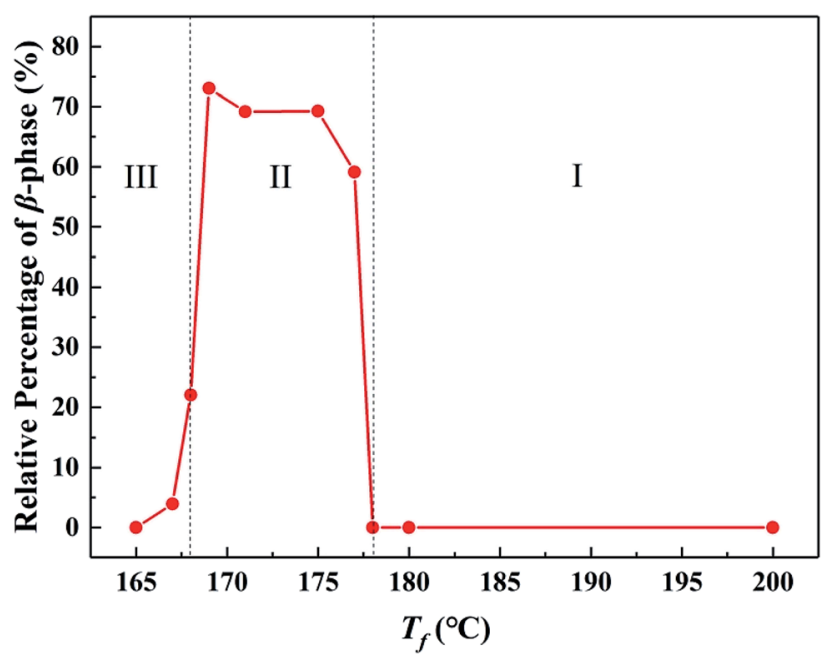

Fig. 1 Variations of relative content of $\beta$-phase $\beta_{c}$ as a function of $T_{f}$ of iPP/GO/WBG-II (GO : WBG- II = $0.5 w t \%: 0.05 w t \%)$. composites. And in Region III $\left(T_{\mathrm{f}}<168{ }^{\circ} \mathrm{C}\right)$, the $\beta_{\mathrm{c}}$ decreased due to the $\alpha$-nucleation effect caused by large crystal nuclei remaining in the melt.

However, the efficiency of OSE might be affected by many other factors. Li et al. ${ }^{25}$ obtained a mesomorphic iPP film by quenching the molten state to $0{ }^{\circ} \mathrm{C}$ in ice-water after erasure of the thermal history at $220^{\circ} \mathrm{C}$ for $10 \mathrm{~min}$. It was found that short melting time favored preservation of the memory effect while a long melting time led to a decrease in the crystallization kinetics at low temperatures. The effect of melting time on the efficiency of OSE was demonstrated. On the other hand, the thermal history of iPP was controlled by tuning the cooling rate during the crystallization process. According to the research, it can be assumed that the OSE efficiency is also affected by the thermal history. For iPP/GO/WBG-II composites with different thermal histories, the influence of melting time on OSE efficiency may also be different.

In previous studies, ${ }^{33-37}$ only the crystallization conditions after the formation of ordered structure were controlled to regulate the efficiency of ordered structure. However, little research has been done on the different initial sources and formation conditions of ordered structure. Therefore, in order to deeply understand the mechanism of ordered structure, we finally focused on the origin and microstructure of ordered structure, which are of great importance. Different thermal history conditions were applied to the iPP/GO/WBG-II composites in order to obtain ordered structures with different morphologies and different interaction efficiencies. By adjusting the optimum initial thermal histories and formation conditions, the OSE could be more effectively used to promote the $\beta$-crystallization and further improve the properties of the composites.

In conclusion, in this paper, the OSEs on $\mathrm{iPP} / \mathrm{GO} / \mathrm{WBG}-\mathrm{II}$ composites with different cooling thermal histories, which crystallized at the slow cooling rate (SLOW), the fast cooling rate (FAST) and the medium cooling rate (MED), respectively, were investigated to discover the influence of crystallization state on OSE. The effects of the melting time $t_{\mathrm{m}}$ on the OSE were also investigated.

\section{Experimental}

\subsection{Materials}

Isotactic polypropylene resin with a trade name of $\mathrm{T} 38 \mathrm{~F}$, molecular weight of 347200 and dispersion index of 3.63, average isotacticity of $97 \%$, melt index of $3.0 \mathrm{~g} / 10 \mathrm{~min}(2.16 \mathrm{~kg}$, $230^{\circ} \mathrm{C}$ ), was purchased from Lanzhou Petroleum Chemical Co, Ltd. (China);

Octadecylamine (ODA), ethanol and xylene were purchased from Chengdu Changzheng Chemical Glass Apparatus Co, Ltd. (China);

Graphene oxide (GO) was purchased from Guangzhou Angstron Graphene Technology Co, Ltd. (China);

The $\beta$-NA with a trade name of WBG-II, composed of heterocyclic double metal (lanthanum and calcium) complexes, was purchased from Guangdong Winner Functional Material Co, Ltd. (China). 


\subsection{Preparation of GO-ODA}

In order to graft ODA on GO, a mature grafting method which has been deeply investigated by previous studies was applied..$^{14,21,38,39}$ The detailed procedures are listed as followed:

GO (0.5 g) and ODA ( $0.9 \mathrm{~g})$ were stirred in $90 \mathrm{~mL}$ ethanol at $90{ }^{\circ} \mathrm{C}$ for $20 \mathrm{~h}$, then vacuum filtered. The obtained powders were ultrasonically dispersed in $100 \mathrm{~mL}$ warm ethanol for $5 \mathrm{~min}$ and vacuum filtered. The rinsing-filtration cycle was repeated four times to remove excess and physisorption of ODA. The resulting ODA grafted GO powder was dried for $24 \mathrm{~h}$ under vacuum at $60{ }^{\circ} \mathrm{C}$. Thus, GO-ODA was successfully prepared.

\subsection{Preparation of iPP/GO/WBG-II composites}

[1] The GO-ODA were ultrasonically dispersed in $200 \mathrm{~mL}$ xylene solution for $30 \mathrm{~min}$. According to the GO content of $5 \mathrm{wt} \%$, a certain amount of iPP was weighed and mixed with GO. The powder was stirred for $2 \mathrm{~h}$ at $130{ }^{\circ} \mathrm{C}$, then filtered and dried for $24 \mathrm{~h}$. The resulting iPP/GO powder was used as masterbatch.

[2] According to GO content of $0.2 \mathrm{~g}$, a certain amount of iPP/ GO sample was weighed in masterbatch. The masterbatch, iPP and WBG-II were mixed in the internal mixer to obtain the iPP/ GO/WBG-II composites with the GO content of $1 \mathrm{wt} \%$ and the WBG-II content of 0.03 wt $\%$. After mixing, the sample was tableted by pressure molding machine.

[3] The pressed samples were used to prepared three samples with different thermal conditions. The first sample was directly placed on the pressure molding machine to crystallize at slow cooling rate (about $1{ }^{\circ} \mathrm{C} \mathrm{min}^{-1}$ ) after holding at $220{ }^{\circ} \mathrm{C}$ and $10 \mathrm{MPa}$ for $10 \mathrm{~min}$ to erase any previous thermal history, which is called SLOW. The second sample was removed from the pressure molding machine after the erasure of the previous thermal history and then immediately placed in ice water $\left(0{ }^{\circ} \mathrm{C}\right)$ to crystallize at fast cooling rate (about $200{ }^{\circ} \mathrm{C} \mathrm{min}^{-1}$ ), which is called FAST. The third sample was removed from the pressure molding machine after elimination of the previous thermal history and then placed at room temperature $\left(25^{\circ} \mathrm{C}\right)$ to crystallize at medium cooling rate (about $10{ }^{\circ} \mathrm{C} \mathrm{min}^{-1}$ ), which is called MED. The thermal treatment program of three samples was shown in Fig. 2.

[4] Finally, DSC and WAXD characterization tests were carried out on the three samples with different cooling thermal histories.
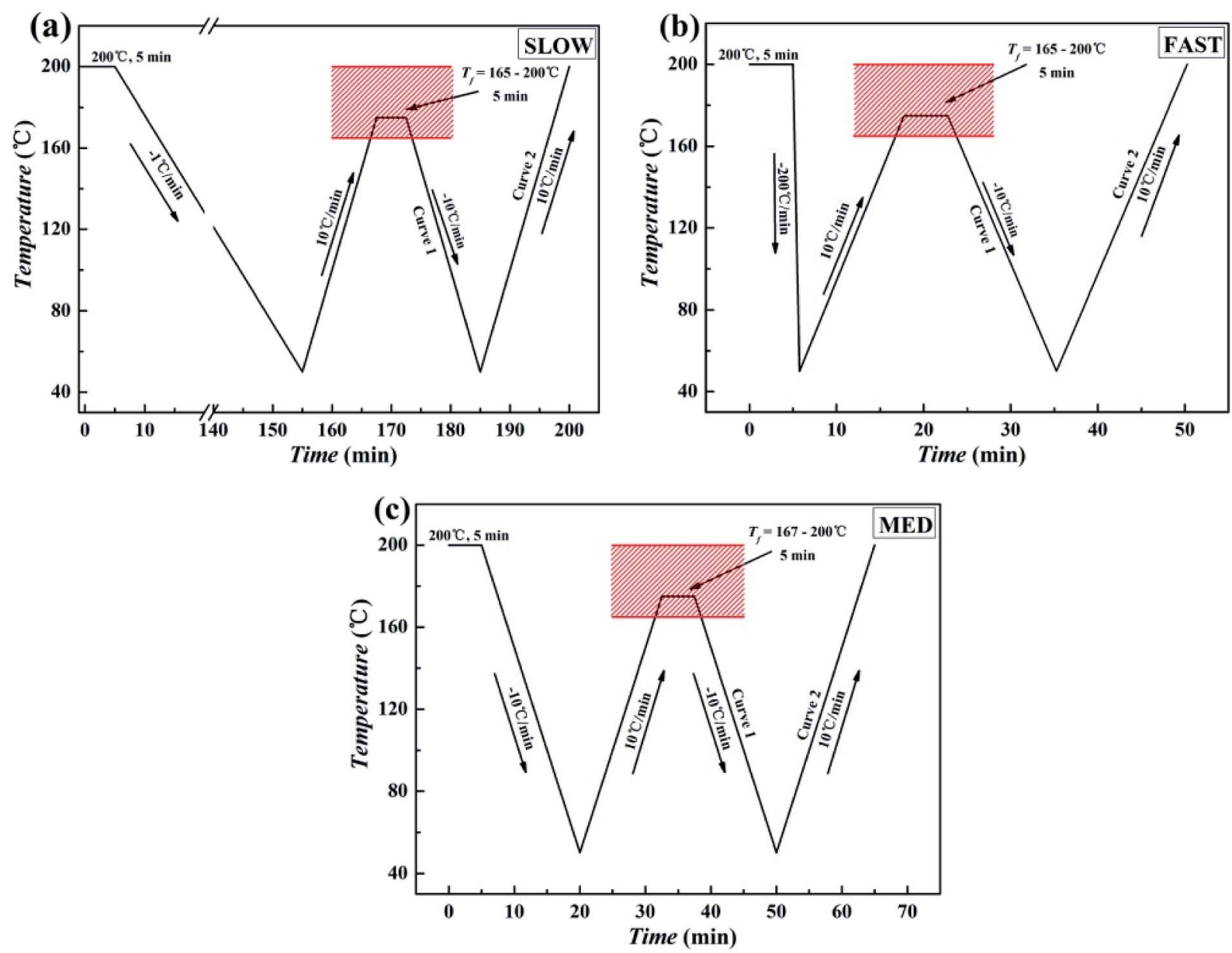

Fig. 2 Illustration of the thermal treatment to study ordered structure effects of iPP/GO/WBG-II composites (GO : WBG-II = 1 wt $\%: 0.03$ wt $\%$ ) with different thermal histories: (a) SLOW; (b) FAST; (c) MED. 


\subsection{Differential scanning calorimetry (DSC)}

All calorimetric experiments were performed with Mettler Toledo DSC1 (Mettler, Switzerland) differential scanning calorimeter under nitrogen atmosphere $\left(50 \mathrm{~mL} \mathrm{~min}^{-1}\right)$. In order to ensure reliability of the data obtained, the temperature scale calibration was performed using indium as a standard. $2-5 \mathrm{mg}$ homogenized sample was used. The thermograms were all fitted by Peakfit 4.12 software according to literatures. ${ }^{16}$ The relative percentage of $\beta$-phase $\left(\beta_{c}\right)$ was estimated by the following equation: ${ }^{40}$

$$
\beta_{\mathrm{c}}=X_{\beta} /\left(X_{\alpha}+X_{\beta}\right)
$$

where $X_{\alpha}$ and $X_{\beta}$ denote the degree of crystallinities of $\alpha$-phase and $\beta$-phase, respectively.

\subsection{Wide-angle X-ray diffraction (WAXD)}

WAXD profiles were recorded with the DX-1000 diffractometer (Dandong Fangyuan Instrument Co., Ltd., China). The wavelength of $\mathrm{Cu} \mathrm{K} \alpha$ was $\lambda=0.154 \mathrm{~nm}$, and the spectra were recorded in the $2 \theta$ range of $5-30^{\circ}$. The scanning rate is $2^{\circ} \mathrm{min}^{-1}$, and the scanning step is $0.02^{\circ}$. The relative percentage of the $\beta$ - phase is determined following standard procedures described in the literature, employing the following expression: ${ }^{41}$

$$
k_{\beta}=\frac{H_{\beta}(110)}{H_{\alpha}(110)+H_{\beta}(110)+H_{\alpha}(040)+H_{\alpha}(130)}
$$

where $k_{\beta}$ denotes the relative content of $\beta$-crystal form (WAXD), $H_{\alpha}(110), H_{\alpha}(040)$, and $H_{\alpha}(130)$ are the intensities of the strongest (110), (040), and (130) diffraction peaks of monoclinic $\alpha$-form, respectively. And $H_{\beta}(110)$ is the intensity of the strongest (110) diffraction peak of the trigonal $\beta$-form. ${ }^{42}$

\subsection{In situ SAXS measurement}

SAXS measurements were performed using a Xeuss SAXS system (Xenocs SA) with $\mathrm{Cu} \mathrm{K} \alpha$ radiation (wavelength $\lambda=0.154 \mathrm{~nm}$ ) equipped with a hot stage. The sample-to-detector distance was $2561 \mathrm{~mm}$. The two-dimensional (2D) SAXS patterns were recorded in every $600 \mathrm{~s}$ by a Mar345 image plate as a detector. Fit2D software package was used to analyze the 2D SAXS patterns. The 2D SAXS patterns were background corrected and then averaged over all directions at constant scattering vector $q$ $(q=(4 \pi \sin \theta) / \lambda$, where $2 \theta$ is the scattering angle), resulting in one-dimensional scattering intensity curves ( $I$ vs. q). The hot
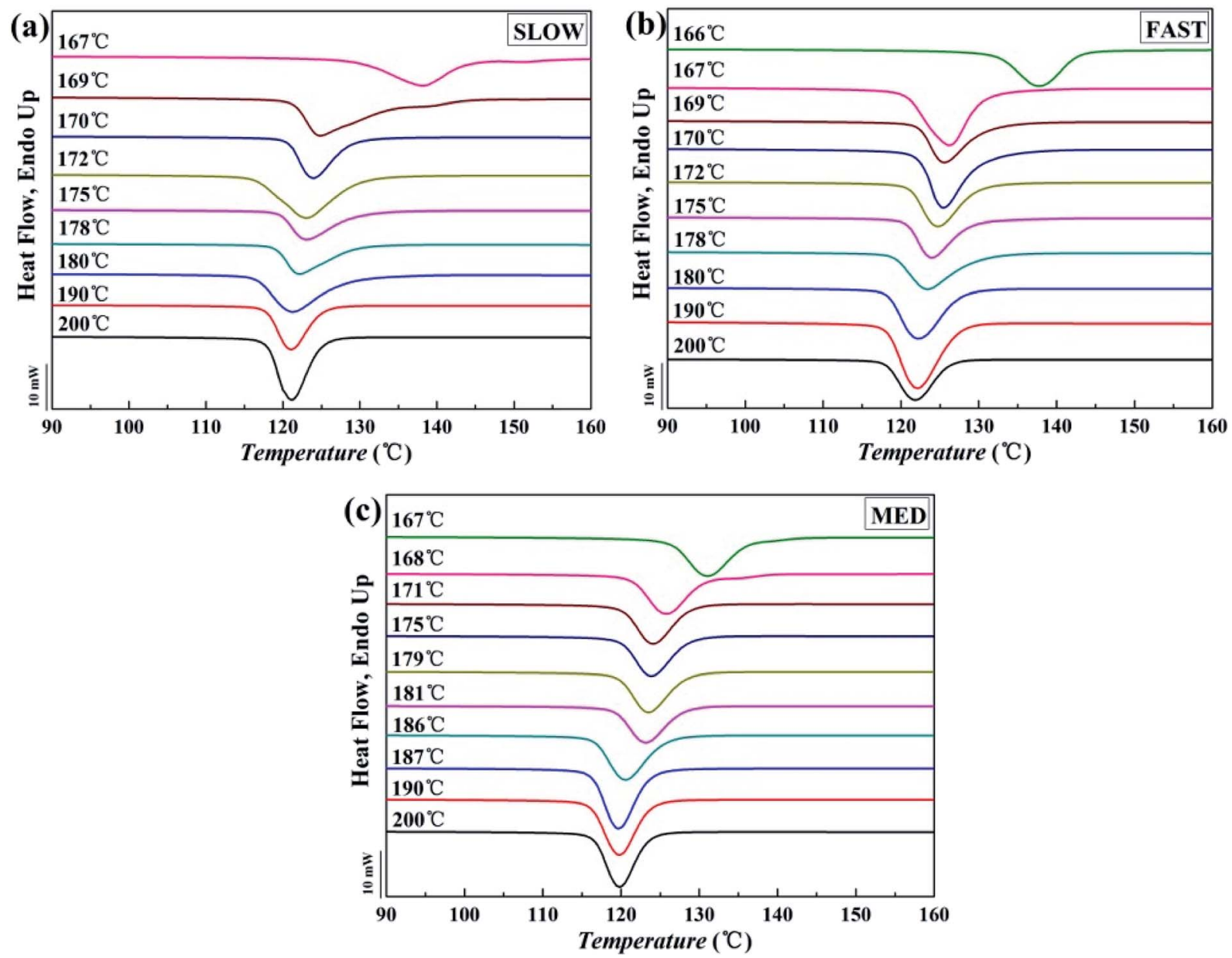

Fig. 3 Crystallization curves of iPP/GO/WBG-II with different thermal histories after held at indicated fusion temperature $\left(T_{f}\right.$, ranging from 166 to $200{ }^{\circ} \mathrm{C}$ ) for 5 min: (a) SLOW, (b) FAST and (c) MED. 
stage was calibrated with an external thermocouple. ${ }^{28}$ In situ SAXS was carried out in a water-cooled hot stage using the following protocols:

[1] Heating the sample from room temperature to $T_{\mathrm{f}}$ at a rate of $5{ }^{\circ} \mathrm{C} \mathrm{min}^{-1}$.

[2] Holding the temperature at $T_{\mathrm{f}}$ for $10 \mathrm{~min}$.

[3] SAXS patterns were taken during melt isothermally at $T_{\mathrm{f}}$.

By the change of the sample-to-detector distance, the Xeuss SAXS system equipped with the hot stage was also used to perform the WAXD testing at high temperatures. The WAXD patterns were recorded in every $200 \mathrm{~s}$.

\section{Results and discussions}

\subsection{Crystallization behaviors}

The DSC crystallization curves are shown in Fig. 3. Fig. 4 intuitively shows how the peak crystallization temperatures $\left(T_{\mathrm{c}}\right)$ change with the variation of $T_{\mathrm{f}}$.

It can be seen that for all the three samples, the entire $T_{\mathrm{f}}$ range can be divided into three regions, which is similar with the case in Fig. 1 . In a high $T_{\mathrm{f}}$ range (Region I), iPP is completely molten and $T_{\mathrm{c}}$ is almost constant. In the medium $T_{\mathrm{f}}$ range (Region II), $T_{\mathrm{c}}$ increases gradually with the decrease of $T_{\mathrm{f}}$, indicating that the $T_{\mathrm{f}}$ is not high enough to make iPP completely molten, and a certain number of ordered structures is retained in the melt. In the low $T_{\mathrm{f}}$ range (Region III), $T_{\mathrm{c}}$ increases rapidly with the decrease of $T_{\mathrm{f}}$ due to the large crystal nucleus remaining in the melt. The result proves that for all samples with different thermal histories, Ordered Structure Effect (OSE) will occur in Region II.

\subsection{Melting behaviors}

The DSC melting curves of the samples are shown in Fig. 5, and 6 shows the variations of the relative percentages of $\beta$-phase $\beta_{\mathrm{c}}$ as a function of $T_{\mathrm{f}}$.

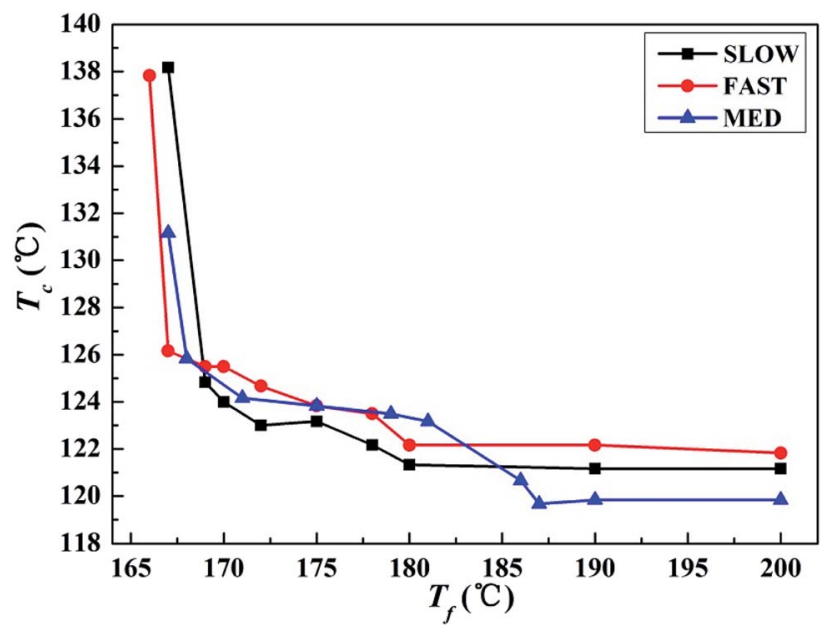

Fig. 4 Variations of the crystallization peak temperature $T_{\mathrm{c}}$ of iPP/GO/ WBG-II with different thermal histories after held at the indicated $T_{f}$ for $5 \mathrm{~min}$, as a function of $T_{\mathrm{f}}$.
In Fig. 5, two types of melting peaks can be observed on the DSC curves: the melting peaks of $\alpha$-phase are located above $155^{\circ} \mathrm{C}$, while those of $\beta$-phase are located below $155^{\circ} \mathrm{C} .{ }^{43}$ It can be seen that for all samples with different thermal histories, as $T_{\mathrm{f}}$ enters OSE regions (Region II of each sample), the characteristic peaks of the $\beta$-phase could be observed clearly, indicating the occurrence of Ordered Structure Effect (OSE). As $T_{\mathrm{f}}$ is too high or too low (Region I or Region III), only characteristic peaks of the $\alpha$-phase can be observed.

On the other hand, it is obvious that the $\beta_{\mathrm{c}}$ of MED is the highest, indicating the highest efficiency of OSE. On the contrary, the $\beta_{\mathrm{c}}$ of SLOW is the lowest, reflecting the lowest efficiency of OSE in enhancing the $\beta$-phase. Moreover, it can be clearly seen that the OSE region of MED is the widest (168-187 ${ }^{\circ} \mathrm{C}$ ), while the OSE region of SLOW is the narrowest (169-180 $\left.{ }^{\circ} \mathrm{C}\right)$.

The results above show that the OSEs on samples with different thermal histories are quite different. The efficiencies and $T_{\mathrm{f}}$ regions of OSE can be ordered as follows: MED $>$ FAST $>$ SLOW, which are influenced by the variations of thermal history. In order to explore the mechanism of the above phenomena, the initial crystallization parameters of samples with different thermal histories are studied.

\subsection{Crystallization parameters of the samples with different thermal histories}

The DSC melting curves are shown in Fig. 7. Fig. 8 shows the variations of the crystallinities percentages of $\beta$-phase $\beta_{\mathrm{c}}$ as a function of $T_{\mathrm{f}}$.

It can be seen that for all the three samples, compared with $T_{\mathrm{f}}=200{ }^{\circ} \mathrm{C}$, the crystallinity of iPP/GO/WBG-II is higher at $T_{\mathrm{f}}=$ $172{ }^{\circ} \mathrm{C}$, since the occurrence of OSE promotes the crystallization and increases the nucleation density.

More significantly, the crystallinities of SLOW is the highest, due to its highest crystallization temperature and longest crystallization time. In contrast, because of its lowest crystallization temperature and shortest crystallization time, the crystallinities of FAST is the lowest. It was shown that the variations of thermal history conditions will influence the crystallinities.

Fig. 9 shows WAXD profiles of iPP/GO/WBG-II composites with different thermal histories. The relative percentages of $\beta$ phase $\beta_{\mathrm{c}}$, crystallinities and crystal size of iPP/GO/WBG-II are listed in Table 1.

It can be observed that the $\beta_{\mathrm{c}} \mathrm{s}$ with different thermal histories are ordered as follows: MED > FAST > SLOW, and the crystallinities with different thermal histories are ordered as follows: SLOW > MED > FAST, which are the same as DSC results. Moreover, the average crystal sizes of $\alpha$-phase are ordered as follows: SLOW $>$ MED $>$ FAST, which is also the order of the crystalline perfection. And the crystal sizes of $\beta$-phase are ordered as follows: MED $>$ FAST $>$ SLOW, which is related to the ability to promote the nucleation and growth of $\beta$-phase.

The above results show that the initial crystallization parameters of samples with different thermal histories are quite different. Due to the highest relative content of $\beta_{\mathrm{c}}$ and high crystallinity, the OSE of MED is greatly promoted. On the 

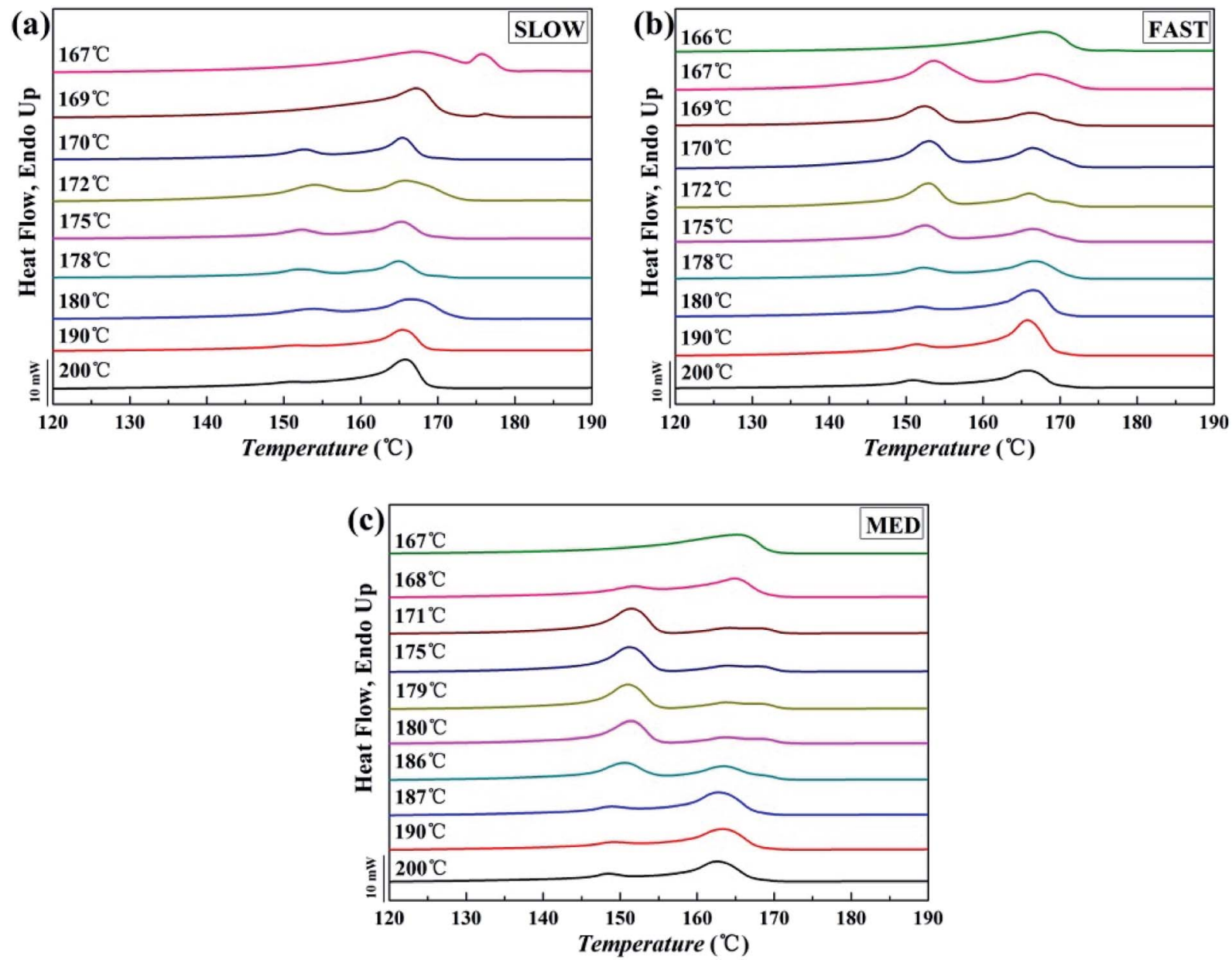

Fig. 5 Melting curves of iPP/GO/WBG-II with different thermal histories after held at the indicated $T_{f}$ for 5 min: (a) SLOW; (b) FAST; (c) MED.

contrary, the OSE of SLOW is inhibited because of the excessive relative content of $\alpha$-phase and the insufficient amount of ordered structure due to the excessive crystalline perfection.

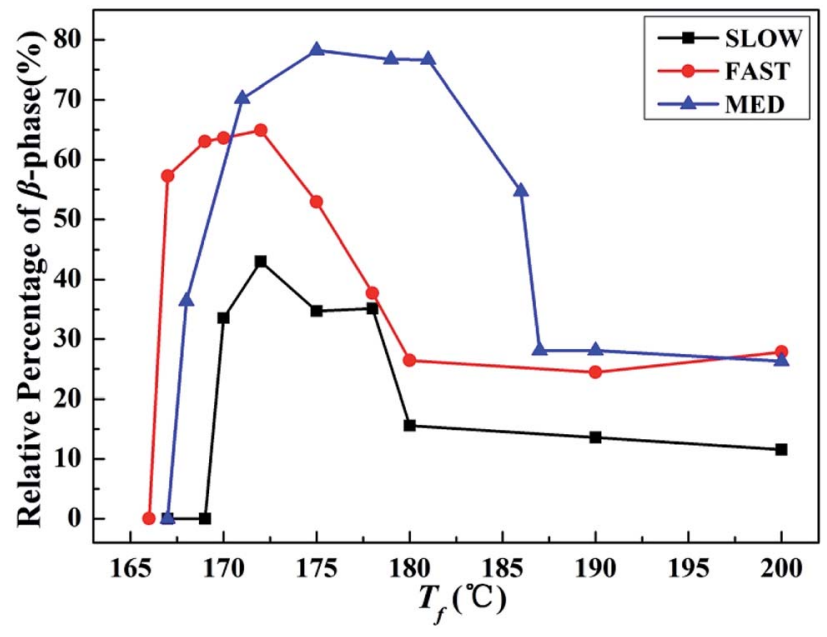

Fig. 6 Relative percentages of $\beta$-phase $\beta_{\mathrm{c}}$ as a function of $T_{\mathrm{f}}$ of iPP/ GO/WBG-II with different thermal histories after held at the indicated $T_{f}$ for $5 \mathrm{~min}$.

\subsection{Influences of melting time at $T_{\mathrm{f}}=172{ }^{\circ} \mathrm{C}$ on OSE}

As can be seen from the results above, due to the excessive crystalline perfection, the crystals were difficult to be melted at lower fusion temperature $T_{\mathrm{f}}$ in a short time (such as $5 \mathrm{~min}$ ), resulting in the suppression of OSE on SLOW. However, if the melting time $t_{\mathrm{m}}$ at lower $T_{\mathrm{f}}$ could be increased so that the overperfect crystal could be melted into the ordered structures, the OSE of SLOW with the highest crystallinity might be greatly promoted.

The comparative experiment was carried out in order to confirm our point of view. The samples called SLOW and FAST were melted at $T_{\mathrm{f}}=172{ }^{\circ} \mathrm{C}\left(T_{\mathrm{f}}\right.$ with the highest OSE efficiency) for different melting time $(2,5,10,20,30,45,60 \mathrm{~min})$, and then DSC tests were carried out to observe the variations of OSE efficiencies.

Fig. 10 shows melting curves after held at $T_{\mathrm{f}}=172{ }^{\circ} \mathrm{C}$ for different melting time. The variations of the relative percentages of $\beta$-phase $\beta_{\mathrm{c}}$ as a function of melting time $t_{\mathrm{m}}$ are shown in Fig. 11.

Obviously, for FAST, as $t_{\mathrm{m}}=5 \mathrm{~min}$, the relative percentage of $\beta$-phase $\beta_{\mathrm{c}}$ reaches the maximum, which indicates that OSE efficiency is the highest at this point. With the increase of the melting time $t_{\mathrm{m}}$, the $\beta_{\mathrm{c}}$ decreases rapidly, which also indicates 

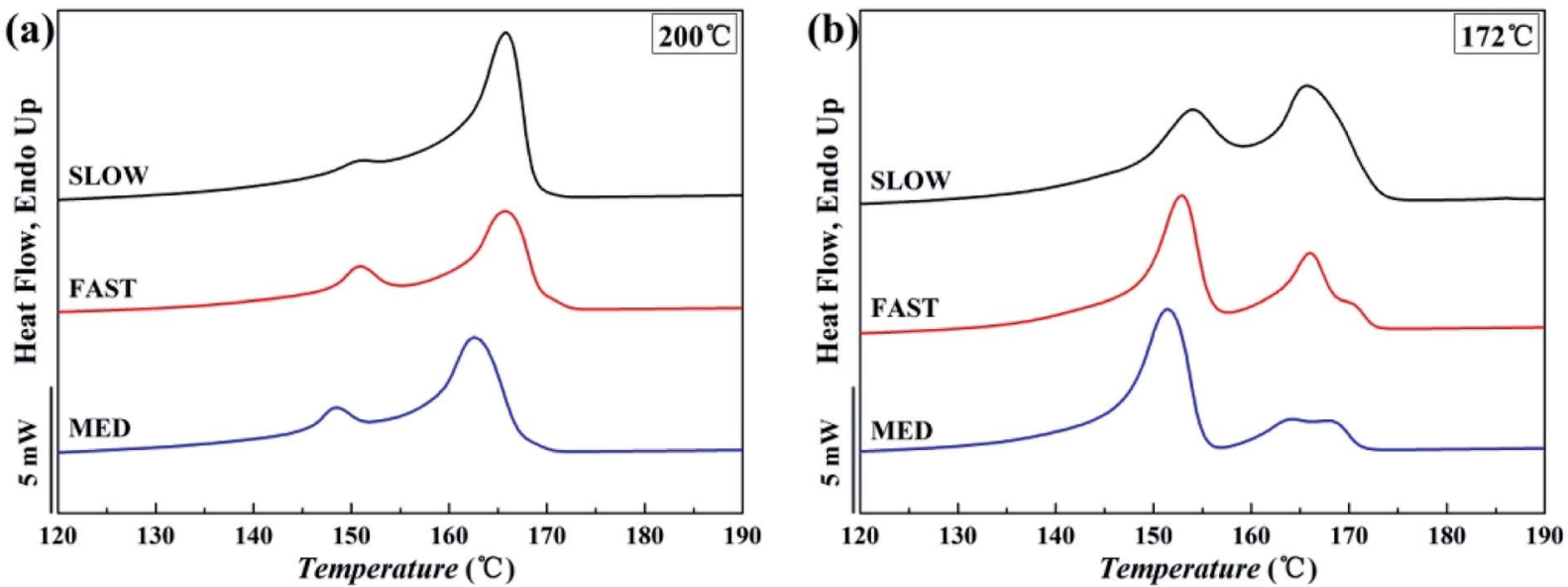

Fig. 7 Melting curves of iPP/GO/WBG-II composites with different thermal histories after held at (a) $T_{\mathrm{f}}=200^{\circ} \mathrm{C}$ and (b) $T_{\mathrm{f}}=172{ }^{\circ} \mathrm{C}$ for 5 min.

the decrease of OSE efficiency. But for SLOW, the $\beta_{\mathrm{c}}$ reaches its maximum value as $t_{\mathrm{m}}=20 \mathrm{~min}$. And the variation rates of OSE efficiency with the $t_{\mathrm{m}}$ are slower than those of FAST. However, no matter how long the $t_{\mathrm{m}}$ is, the $\beta_{\mathrm{c}}$ of SLOW is lower than that of FAST.

In order to explain the mechanism of above phenomenon, Fig. 12 shows the possible structural changes that occur during the melting process of iPP/GO/WBG-II. For FAST, due to its lower crystallinity and lower crystalline perfection, the iPP/GO/ WBG-II crystal will be completely melted in a short time $(t=5$ min), and completely converted to the pre-ordered structure. At this point, the amount of ordered structures reaches the maximum, leading to the highest efficiency of OSE. However, with the further increase of melting time, the ordered structure will also be gradually melted. As the amount of ordered structure decreases, OSE efficiency drops rapidly. On the contrary, for SLOW, due to its higher crystallinity and higher crystalline perfection, the iPP/GO/WBG-II crystal cannot completely melted in a short time, and the transformation of the crystal to the preordered structure is limited. However, with the increase of

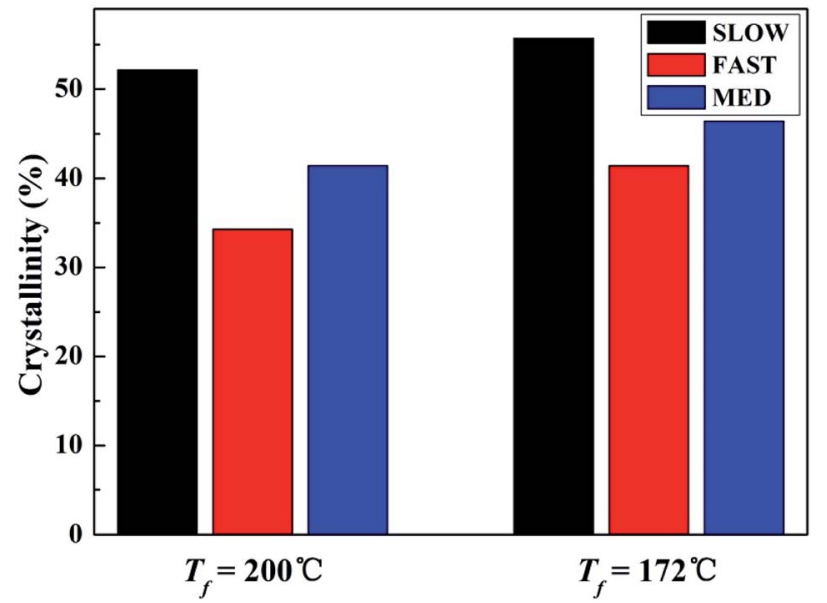

Fig. 8 Crystallinities of iPP/GO/WBG-II with different thermal histories after held at $T_{f}=200^{\circ} \mathrm{C}$ and $T_{f}=172^{\circ} \mathrm{C}$ for $5 \mathrm{~min}$. melting time, the crystal will eventually be completely melted, leading to the improvement of the amount of ordered structure and the OSE efficiency. Similarly, in a sufficiently long melting time ( $t=60 \mathrm{~min})$, the ordered structure will also be eventually melted completely, resulting in the absence of OSE.

\subsection{In situ SAXS measurement}

As mentioned above, the thermal history of the sample greatly influences the occurrence of OSE behavior, i.e., the $\alpha$-/ $\beta$-polymorphic selectivity of the ordered structure. Therefore, it is speculated that the ordered structures of iPP/GO/WBG-II composites with varying thermal histories may have different physical status at the given $T_{\mathrm{f}}$. In this section, in situ SAXS technique has been used as a powerful tool to investigate the crystallization behavior at the early stage. Considering that after hold at $T_{\mathrm{f}}=178{ }^{\circ} \mathrm{C}$ or $T_{\mathrm{f}}=187^{\circ} \mathrm{C}$, the $\beta_{\mathrm{c}}$ values of FAST, MED and SLOW in the subsequent melting were quite different from each other $(37.7 \%, 77.0 \%$ and $35.1 \%)$ respectively at $T_{\mathrm{f}}=178{ }^{\circ} \mathrm{C}$; while at $T_{\mathrm{f}}=187^{\circ} \mathrm{C}$ the OSE only takes place in MED, reflecting

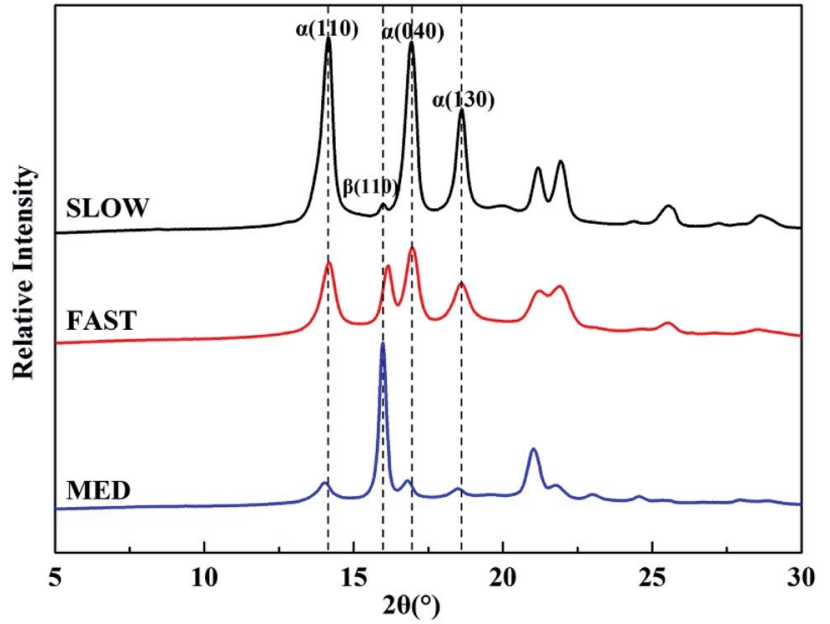

Fig. 9 WAXD profiles of iPP/GO/WBG-II with different thermal histories. 
Table 1 The relative percentages of $\beta$-phase $\beta_{c}$, crystallinities and crystal size of iPP/GO/WBG-II

\begin{tabular}{|c|c|c|c|c|c|c|c|c|}
\hline \multirow[b]{2}{*}{ Sample } & \multirow[b]{2}{*}{$\beta_{\mathrm{c}}(\%)$} & \multirow[b]{2}{*}{$\alpha_{\mathrm{c}}(\%)$} & \multirow[b]{2}{*}{ Amorphous (\%) } & \multirow[b]{2}{*}{ Crystallinity (\%) } & \multicolumn{4}{|c|}{ Crystalline sizes $L(\mathrm{~nm})$} \\
\hline & & & & & $\alpha(110)$ & $\alpha(130)$ & $\alpha(040)$ & $\beta(110)$ \\
\hline SLOW & 7.5 & 92.5 & 46.7 & 53.3 & 19.0 & 20.4 & 22.3 & 12.7 \\
\hline FAST & 26.0 & 74.0 & 59.5 & 40.5 & 15.8 & 17.8 & 7.3 & 24.6 \\
\hline MED & 65.0 & 35.0 & 53.2 & 46.8 & 10.8 & 18.2 & 17.8 & 32.9 \\
\hline
\end{tabular}
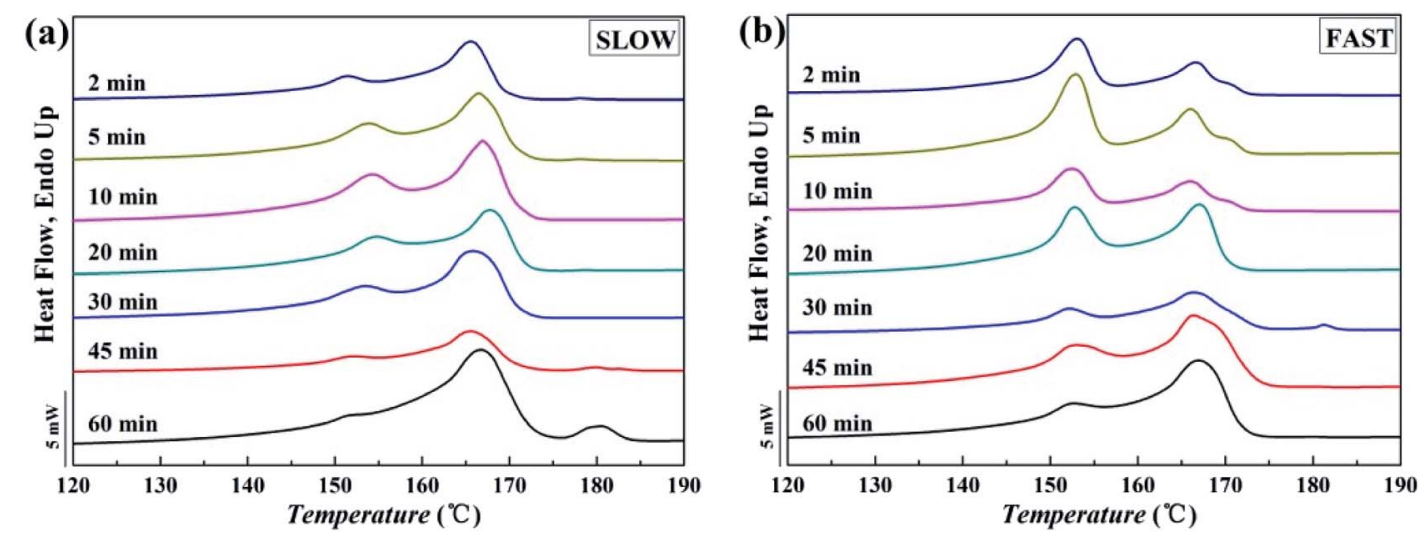

Fig. 10 Melting curves of iPP/GO/WBG-II with different thermal histories after held at $T_{\mathrm{f}}=172{ }^{\circ} \mathrm{C}$ for different melting time: (a) SLOW; (b) FAST.

that they may have very different ordered structure status, in this section, in situ SAXS was performed on the three samples at the fusion temperatures of $T_{\mathrm{f}}=178^{\circ} \mathrm{C}$ and $187^{\circ} \mathrm{C}$. Results were analyzed using the method reported in previous study, ${ }^{\mathbf{4 4}}$ and the integrated scattered intensity profiles of the samples are shown in Fig. 13, which reflects the distribution of the heterogeneity in the samples.

At $T_{\mathrm{f}}=178{ }^{\circ} \mathrm{C}$, the DSC results indicate some ordered structures survive in iPP melt. For all the samples, although there is no visible Bragg peak in the $I-q$ curves (only scattering

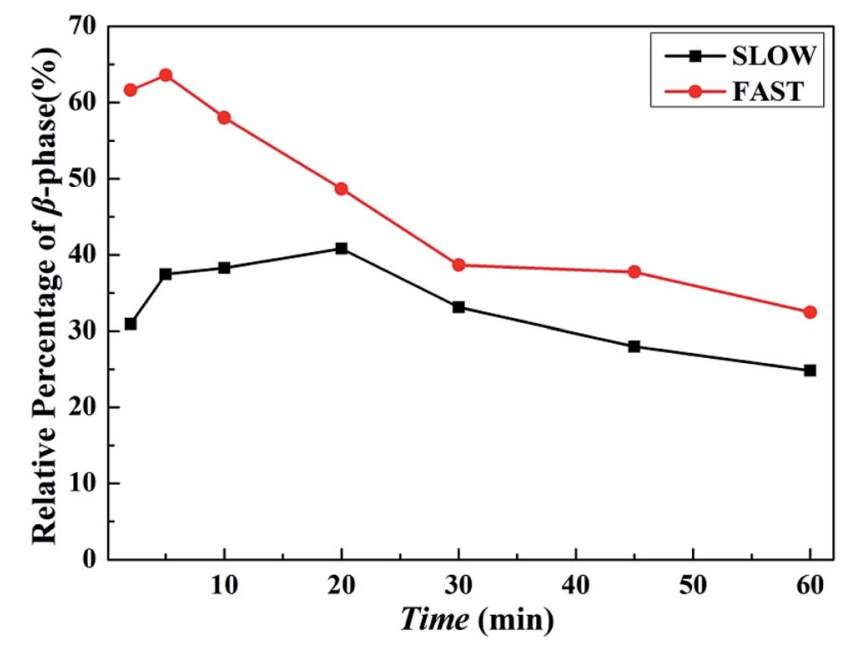

Fig. 11 Relative percentages of $\beta$-phase $\beta_{c}$ as a function of melting time $t_{m}$ of iPP/GO/WBG-II with different thermal histories after held at $T_{\mathrm{f}}=172{ }^{\circ} \mathrm{C}$ for different melting time. bumps can be seen at $q=0.1-0.2$ ), the remnants of crystalline scattering peaks in the plots of Lorentz corrected SAXS intensity can be clearly seen, indicating the existence of lamellar structures at $T_{\mathrm{f}}=178{ }^{\circ} \mathrm{C}$ for all the samples. Moreover, the SAXS intensity profile of MED is obviously different from those of FAST and SLOW, indicating the significant differences between their ordered structures: the ordered structures of FAST and SLOW are quite similar from each other, while MED exhibits a different ordered structure status at $T_{\mathrm{f}}=178^{\circ} \mathrm{C}$.

On the other hand, in Fig. $13(\mathrm{~b}), T_{\mathrm{f}}=187^{\circ} \mathrm{C}$ (in Region I for FAST and SLOW, while Region II for MED), the SAXS intensities of all the samples are much lower than that at $T_{\mathrm{f}}=178{ }^{\circ} \mathrm{C}$ and there is no Bragg peak. Since there is no scattering bump in the curves, Bragg peak cannot appear in Lorentz corrected scattering curve. It indicates that the survived periodic crystalline lamellar structures have melted. However, we still deduce that some ordered structures survive at such a high temperature from the scattering intensities of the samples. Considering that scattering experiments in fractals with X-rays have shown that the scattering intensity follows a power law as $I \propto q^{-\alpha}$, values of $\alpha=1,2,3$ are expected when the scattering objects are rods, discs and spheres respectively. Evidently, for MED, $\alpha=2$, indicating its ordered structures with high $\beta$-nucleation efficiency are flat disks. ${ }^{45}$ For FAST and SLOW, $\alpha=2.3$ and 2.2 respectively, corresponding to ordered structures without high $\beta$-nucleation efficiency in a disk with convoluted surface. ${ }^{45}$

SAXS results show that the ordered structures of FAST and SLOW are quite similar at $178^{\circ} \mathrm{C}$ and $187^{\circ} \mathrm{C}$, while the ordered structure of MED is quite different. Associated with the fact that the initial thermal histories result in different contents of $\beta$ phase $(65.0 \%$ for MED, only $26.0 \%$ and $7.5 \%$ for FAST and 


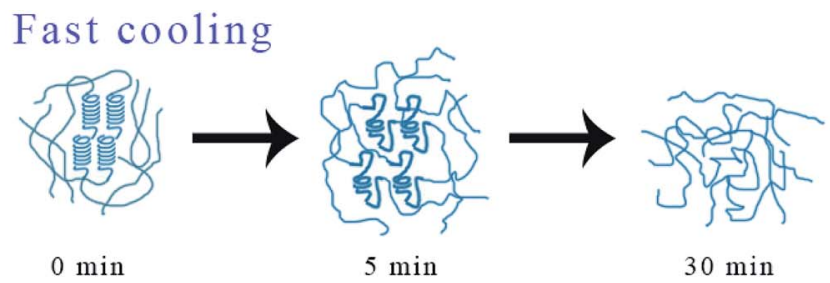

Slow cooling

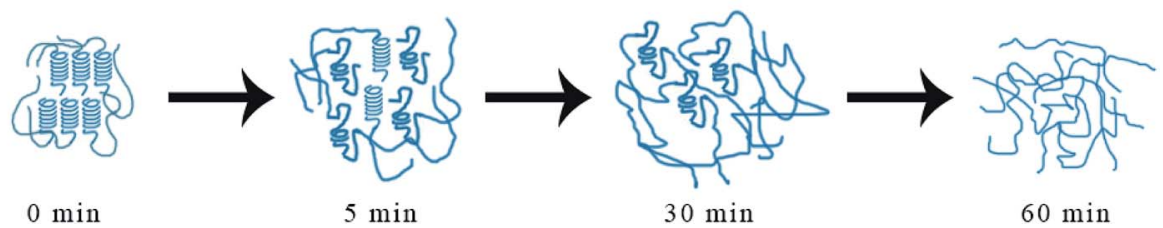

Fig. 12 Possible structural changes in the iPP/GO/WBG-II melt with different thermal histories during melting at $172{ }^{\circ} \mathrm{C}$.
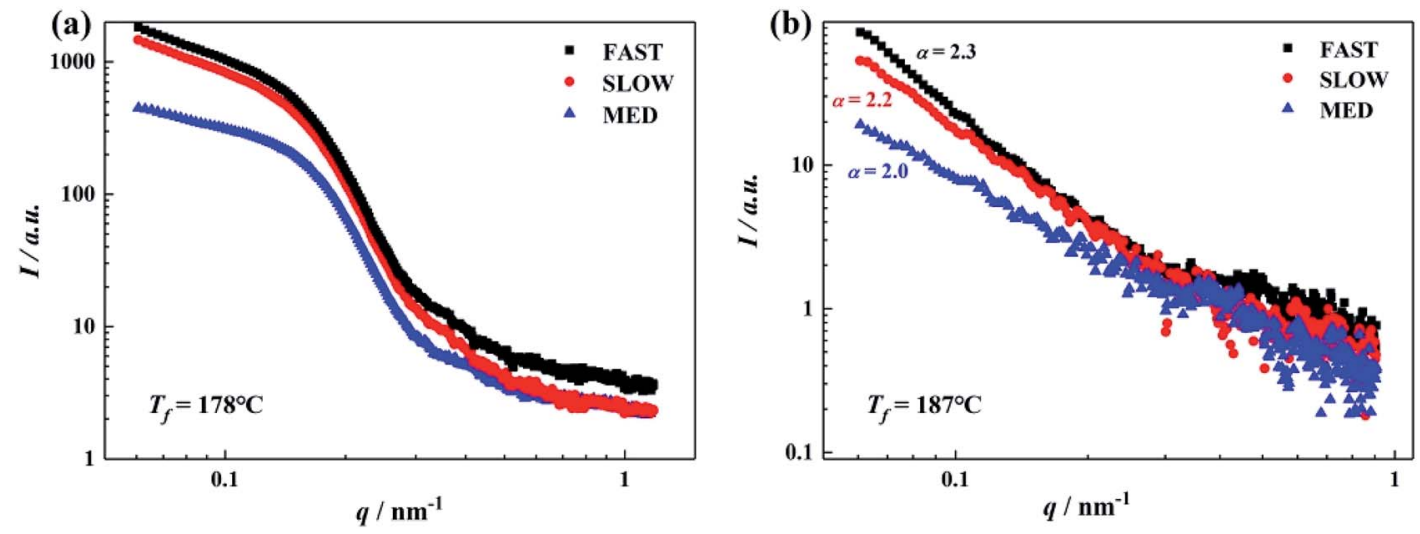

Fig. 13 SAXS intensity profiles of iPP/GO/WBG-II obtained after isothermal melting at the various $T_{\mathrm{f}}$ : (a) $178{ }^{\circ} \mathrm{C}$; (b) $187^{\circ} \mathrm{C}$.

SLOW), it can be inferred that the initial content of $\beta$-phase has a great influence on the OSE behavior of the samples, which might be the reason why the OSE behavior of MED is different from that of FAST and SLOW. The higher initial content of $\beta$ phase is conducive to the formation of ordered structures with higher OSE efficiency.

\section{Conclusions}

In this paper, the Ordered Structure Effects (OSE) of the iPP/GO/ WBG-II (GO : WBG-II = 1 wt\%: 0.03 wt $\%$ ) with different thermal histories, which crystallized at slow cooling rate (called SLOW), fast cooling rate (called FAST) and medium cooling rate (called MED), respectively, was investigated. The ordered structure status was controlled by tuning the fusion temperature $T_{\mathrm{f}}$ and melting time $t_{\mathrm{m}}$. The conclusions can be drawn as following:

For all samples, the entire $T_{\mathrm{f}}$ range could be divided into three regions, and OSE would occur in an appropriate $T_{\mathrm{f}}$ region (Region II). The relative percentages of $\beta$-phase $\beta_{\mathrm{c}}$ of MED was the highest, while that of SLOW was the lowest. It also represented the order of OSE efficiency. Analyzing the initial state of crystallization, it could be found that the crystallinity and crystalline perfection of SLOW were the highest, while that of FAST was the lowest. As $T_{\mathrm{f}}=172{ }^{\circ} \mathrm{C}$, the $\beta_{\mathrm{c}}$ of FAST reached the maximum at $t_{\mathrm{m}}=5 \mathrm{~min}$, while that of SLOW reached the maximum at $t_{\mathrm{m}}=20 \mathrm{~min}$. The OSE efficiencies of both first increased and then decreased.

The morphology of ordered structure and the OSE efficiency were affected by the thermal history, and it could be improved by selecting the appropriate $t_{\mathrm{m}}$. Moreover, the initial content of $\beta$-phase has a great influence on the OSE behavior. The higher initial content of $\beta$-phase is conducive to the formation of ordered structure with higher OSE efficiency.

\section{Conflicts of interest}

There are no conflicts to declare.

\section{Acknowledgements}

We gratefully acknowledge the National Natural Science Foundation of China (NSFC 51503134, 51721091, 51702282), the State Key Laboratory of Polymer Materials Engineering (Grant No. SKLPME 2017-3-02) and the Key Industry Technology 
Innovation Projects of Chongqing (CSTC2017zdcy-zdyf0297) for the financial support.

\section{References}

1 C. Lee, X. Wei, J. W. Kysar and J. Hone, Measurement of the elastic properties and intrinsic strength of monolayer graphene, Science, 2008, 321(5887), 385-388.

2 S. Dutta and S. K. Pati, Novel properties of graphene nanoribbons: a review, J. Mater. Chem., 2010, 20(38), 8207.

3 Y. Cui, S. I. Kundalwal and S. Kumar, Gas barrier performance of graphene/polymer nanocomposites, Carbon, 2016, 98, 313-333.

4 T. Liu, K. Yu, L. Gao, H. Chen, N. Wang, L. Hao, T. Li, H. He and Z. Guo, A graphene quantum dot decorated $\mathrm{SrRuO}_{3}$ mesoporous film as an efficient counter electrode for highperformance dye-sensitized solar cells, J. Mater. Chem. A, 2017, 5(34), 17848-17855.

5 O.-K. Park, J.-Y. Hwang, M. Goh, J. H. Lee, B.-C. Ku and N.-H. You, Mechanically Strong and Multifunctional Polyimide Nanocomposites Using Amimophenyl Functionalized Graphene Nanosheets, Macromolecules, 2013, 46(9), 3505-3511.

6 V. Goyal and A. A. Balandin, Thermal properties of the hybrid graphene-metal nano-micro-composites: applications in thermal interface materials, Appl. Phys. Lett., 2012, 100(7), 073113.

7 L. Dashairya, M. Rout and P. Saha, Reduced graphene oxidecoated cotton as an efficient absorbent in oil-water separation, Adv. Compos. Hybrid Mater., 2017, 1(1), 135-148.

8 G. Yu, Y. Lu, J. Guo, M. Patel, A. Bafana, X. Wang, B. Qiu, C. Jeffryes, S. Wei, Z. Guo and E. K. Wujcik, Carbon nanotubes, graphene, and their derivatives for heavy metal removal, Adv. Compos. Hybrid Mater., 2017, 1(1), 56-78.

9 T. Su, Q. Shao, Z. Qin, Z. Guo and Z. Wu, Role of Interfaces in Two-Dimensional Photocatalyst for Water Splitting, ACS Catal., 2018, 8(3), 2253-2276.

10 Y. Cao, J. Feng and P. Wu, Polypropylene-grafted graphene oxide sheets as multifunctional compatibilizers for polyolefin-based polymer blends, J. Mater. Chem., 2012, 22(30), 14997.

11 J. R. Potts, S. H. Lee, T. M. Alam, J. An, M. D. Stoller, R. D. Piner and R. S. Ruoff, Thermomechanical properties of chemically modified graphene/poly(methyl methacrylate) composites made by in situ polymerization, Carbon, 2011, 49(8), 2615-2623.

12 J. Liang, Y. Huang, L. Zhang, Y. Wang, Y. Ma, T. Guo and Y. Chen, Molecular-Level Dispersion of Graphene into Poly(vinyl alcohol) and Effective Reinforcement of their Nanocomposites, Adv. Funct. Mater., 2009, 19(14), 22972302.

$13 \mathrm{Z}$. $\mathrm{Xu}$ and C. Gao, In situ Polymerization Approach to Graphene-Reinforced Nylon-6 Composites, Macromolecules, 2010, 43(16), 6716-6723.

14 W. Li, X.-Z. Tang, H.-B. Zhang, Z.-G. Jiang, Z.-Z. Yu, X.-S. Du and Y.-W. Mai, Simultaneous surface functionalization and reduction of graphene oxide with octadecylamine for electrically conductive polystyrene composites, Carbon, 2011, 49(14), 4724-4730.

15 Y. S. Yun, Y. H. Bae, D. H. Kim, J. Y. Lee, I.-J. Chin and H.-J. Jin, Reinforcing effects of adding alkylated graphene oxide to polypropylene, Carbon, 2011, 49(11), 3553-3559.

16 J. Kang, F. Yang, T. Wu, H. Li, Y. Cao and M. Xiang, Polymerization control and fast characterization of the stereo-defect distribution of heterogeneous Ziegler-Natta isotactic polypropylene, Eur. Polym. J., 2012, 48(2), 425-434.

17 S. Zhou, W. Wang, S. Zhao, Z. Xin and Y. Shi, Shear-induced $\beta$-form polypropylene in long chain branching isotactic polypropylene, Polym. Eng. Sci., 2016, 56(2), 240-247.

18 X. Cao, Y. Qiao, Y. Chen, G. He and H. Lin, Critical role of depressurization and effects of saturation conditions in the formation of $\beta$-crystal during isotactic polypropylene foaming with supercritical $\mathrm{CO}_{2}$, Polym. Eng. Sci., 2016, 56(9), 980-986.

19 J. Kang, B. Wang, H. Peng, J. Li, J. Chen, J. Gai, Y. Cao, H. Li, F. Yang and M. Xiang, Investigation on the dynamic crystallization and melting behavior of $\beta$-nucleated isotactic polypropylene with different stereo-defect distribution-the role of dual-selective $\beta$-nucleation agent, Polym. Adv. Technol., 2014, 25(1), 97-107.

20 H. Wang, P. G. Ren, Y. H. Chen, D. X. Yan, Z. M. Li and L. Xu, Effects of Dodecyl Amine Functionalized Graphene Oxide on the Crystallization Behavior of Isotactic Polypropylene, $J$. Appl. Polym. Sci., 2014, 131(6).

21 R.-Y. Bao, J. Cao, Z.-Y. Liu, W. Yang, B.-H. Xie and M.-B. Yang, Towards balanced strength and toughness improvement of isotactic polypropylene nanocomposites by surface functionalized graphene oxide, J. Mater. Chem. A, 2014, 2(9), 3190-3199.

22 F. Su, X. Li, W. Zhou, S. Zhu, Y. Ji, Z. Wang, Z. Qi and L. Li, Direct Formation of Isotactic Poly(1-butene) Form I Crystal from Memorized Ordered Melt, Macromolecules, 2013, 46(18), 7399-7405.

23 D. Cavallo, L. Gardella, G. Portale, A. J. Müller and G. C. Alfonso, Self-nucleation of isotactic poly(1-butene) in the trigonal modification, Polymer, 2014, 55(1), 137-142.

24 J. Kang, H. Peng, B. Wang, J. Chen, F. Yang, Y. Cao, H. Li and M. Xiang, Investigation on the Self-nucleation Behavior of Controlled-rheology Polypropylene, J. Macromol. Sci., Part B: Phys., 2015, 54(2), 127-142.

25 X. Li, F. Su, Y. Ji, N. Tian, J. Lu, Z. Wang, Z. Qi and L. Li, Influence of the memory effect of a mesomorphic isotactic polypropylene melt on crystallization behavior, Soft Matter, 2013, 9(35), 8579.

26 Q. Liu, X. Sun, H. Li and S. Yan, Orientation-induced crystallization of isotactic polypropylene, Polymer, 2013, 54(17), 4404-4421.

27 D. Cavallo, G. Portale, L. Balzano, F. Azzurri, W. Bras, G. W. Peters and G. C. Alfonso, Real-Time WAXD Detection of Mesophase Development during Quenching of Propene/ Ethylene Copolymers, Macromolecules, 2010, 43(24), 1020810212. 
28 B. Zhang, J. Chen, F. Ji, X. Zhang, G. Zheng and C. Shen, Effects of melt structure on shear-induced $\beta$-cylindrites of isotactic polypropylene, Polymer, 2012, 53(8), 1791-1800.

29 Y. Qin, Y. Xu, L. Zhang, G. Zheng, K. Dai, C. Liu, X. Yan, J. Guo and Z. Guo, Shear-induced interfacial sheath structure in isotactic polypropylene/glass fiber composites, Polymer, 2015, 70, 326-335.

30 Y. Qin, Y. Xu, L. Zhang, G. Zheng, X. Yan, K. Dai, C. Liu, C. Shen and Z. Guo, Interfacial interaction enhancement by shear-induced $\beta$-cylindrite in isotactic polypropylene/ glass fiber composites, Polymer, 2016, 100, 111-118.

31 C. Ji, M. Xie, B. Chang, K. Dai, B. Wang, G. Zheng, C. Liu and C. Shen, $\beta$-Crystal in injection moulded poly(ethylene terephthalate) fibre/isotactic polypropylene composite, Composites, Part A, 2013, 46, 26-33.

32 B. Chang, M. Xie, K. Dai, G. Zheng, S. Wang, C. Liu, J. Chen and C. Shen, Pre-shear induced anomalous distribution of $\beta$ form in injection molded iPP, Polym. Test., 2013, 32(3), 545552.

33 Y. Yu, F. Zeng, J. Chen, J. Kang, F. Yang, Y. Cao and M. Xiang, Regulating polycrystalline behavior of the $\beta$-nucleated isotactic polypropylene/graphene oxide composites by melt memory effect, Polym. Compos., 2019, 40(S1), E440-E448.

34 Y. Yu, F. Zeng, J. Chen, J. Kang, F. Yang, Y. Cao and M. Xiang, Isothermal crystallization kinetics and subsequent melting behavior of $\beta$-nucleated isotactic polypropylene/graphene oxide composites with different ordered structure, Polym. Int., 2018, 67(9), 1212-1220.

35 Q. Zhang, H. Peng, J. Kang, Y. Cao and M. Xiang, Effects of melt structure on non-isothermal crystallization behavior of isotactic polypropylene nucleated with $\alpha / \beta$ compounded nucleating agents, Polym. Eng. Sci., 2017, 57(9), 989-997.

36 Q. Zhang, Z. Chen, B. Wang, J. Chen, F. Yang, J. Kang, Y. Cao, M. Xiang and H. Li, Effects of melt structure on crystallization behavior of isotactic polypropylene nucleated with $\alpha / \beta$ compounded nucleating agents, J. Appl. Polym. Sci., 2015, 132(4) , 41355.
37 Y. Yu, F. Zeng, J. Chen, J. Kang, F. Yang, Y. Cao and M. Xiang, Effects of ordered structure on non-isothermal crystallization kinetics and subsequent melting behavior of $\beta$-nucleated isotactic polypropylene/graphene oxide composites, J. Therm. Anal. Calorim., 2018, 136(4), 16671678.

38 G. Wang, X. Shen, B. Wang, J. Yao and J. Park, Synthesis and characterisation of hydrophilic and organophilic graphene nanosheets, Carbon, 2009, 47(5), 1359-1364.

39 Z. Lin, Y. Liu and C. P. Wong, Facile fabrication of superhydrophobic octadecylamine-functionalized graphite oxide film, Langmuir, 2010, 26(20), 16110-16114.

40 H. Peng, B. Wang, J. Gai, J. Chen, F. Yang, Y. Cao, H. Li, J. Kang and M. Xiang, Investigation on the morphology and tensile behavior of $\beta$-nucleated isotactic polypropylene with different stereo-defect distribution, J. Appl. Polym. Sci., 2014, 131(6, ), 40027.

$41 \mathrm{Q}$. $\mathrm{Lu}$ and Q. Dou, $\beta$-Crystal formation of isotactic polypropylene induced by N, N'-dicyclohexylsuccinamide, $J$. Polym. Res., 2008, 16(5), 555-560.

42 D. L. Dorset, M. P. McCourt, S. Kopp, M. Schumacher, T. Okihara and B. Lotz, Isotactic polypropylene, $\beta$-phase: a study in frustration, Polymer, 1998, 39(25), 6331-6337.

43 Z. Horvath, I. E. Sajo, K. Stoll, A. Menyhard and J. Varga, The effect of molecular mass on the polymorphism and crystalline structure of isotactic polypropylene, eXPRESS Polym. Lett., 2010, 4(2), 101-114.

44 J. Kang, Z. Chen, F. Yang, J. Chen, Y. Cao, G. Weng and M. Xiang, Understanding the effects of nucleating agent concentration on the polymorphic behavior of $\beta$-nucleated isotactic polypropylene with different melt structures, Colloid Polym. Sci., 2015, 293(7), 2061-2073.

45 D. G. Papageorgiou, G. Z. Papageorgiou, E. Zhuravlev, D. Bikiaris, C. Schick and K. Chrissafis, Competitive Crystallization of a Propylene/Ethylene Random Copolymer Filled with a $\beta$-Nucleating Agent and Multi-Walled Carbon Nanotubes. Conventional and Ultrafast DSC Study, J. Phys. Chem. B, 2013, 117(47), 14875-14884. 